\title{
Domination Numbers of Amalgamations of Cycles at Connected Subgraphs
}

\author{
Prakassawat Boonmee $\mathbb{D}^{1},{ }^{1}$ Jirapa Ma - In $\mathbb{D}^{2},{ }^{2}$ and Sayan Panma $\mathbb{D}^{2,3}$ \\ ${ }^{1}$ Department of Mathematics, Faculty of Science and Technology, Muban Chom Bueng Rajabhat University, \\ Chom Bueng, Ratchaburi, Thailand \\ ${ }^{2}$ Department of Mathematics, Faculty of Science, Chiang Mai University, Chiang Mai, Thailand \\ ${ }^{3}$ Advanced Research Center for Computational Simulation, Department of Mathematics, Faculty of Science, \\ Chiang Mai University, Chiang Mai, Thailand
}

Correspondence should be addressed to Sayan Panma; panmayan@yahoo.com

Received 16 April 2021; Revised 10 November 2021; Accepted 2 December 2021; Published 28 January 2022

Academic Editor: Shaofang Hong

Copyright (c) 2022 Prakassawat Boonmee et al. This is an open access article distributed under the Creative Commons Attribution License, which permits unrestricted use, distribution, and reproduction in any medium, provided the original work is properly cited. A set $S$ of vertices of a graph $G$ is a dominating set of $G$ if every vertex in $V(G)$ is adjacent to some vertex in $S$. A minimum
dominating set in a graph $G$ is a dominating set of minimum cardinality. The cardinality of a minimum dominating set is called the
domination number of $G$ and is denoted by $\gamma(G)$. Let $G_{1}$ and $G_{2}$ be disjoint graphs, $H_{1}$ be a subgraph of $G_{1}, H_{2}$ be a subgraph of
$G_{2}$, and $f$ be an isomorphism from $H_{1}$ to $H_{2}$. The amalgamation (the glued graph) of $G_{1}$ and $G_{2}$ at $H_{1}$ and $H_{2}$ with respect to $f$ is
the graph $G=G_{1} \triangleleft \triangleright G_{2}$ obtained by forming the disjoint union of $G_{1}$ and $G_{2}$ and then identifying $H_{1}$ and $H_{2}$ with respect to $f$. In
this paper, we determine the domination numbers of the amalgamations of two cycles at connected subgraphs.

\section{Introduction}

Studying on several graph parameters is an interesting topic in graph theory. The domination number is one of the most importance parameter which was introduced from 1958 by Berge [1], called as "coefficient of external stability." In 1962, Ore [2] studied the same concept and used the name "dominating set" and "domination number" for a graph. In 1977, Cockayane and Hedetneimi [3] gave a survey of the results about dominating sets and used the notation $\gamma(G)$ for the domination number of a graph. In 1998, a text devoted to this subject was introduced by Haynes et al. [4]. Over 2000 articles on graph domination numbers have been studied extensively (see, for example, [2, 3, 5-16]), in particular, the study of the domination number of product graphs such as the Cartesian product of two cycles [12], the cross product of two paths [8], and the lexicographic product of two graphs [16]. It is natural to investigate the domination number of the amalgamation of two graphs, especially, the domination number of the amalgamation of two paths or two cycles.

Let $G_{1}$ and $G_{2}$ be disjoint graphs and $H_{1} \subseteq G_{1}$ and $H_{2} \subseteq G_{2}$ such that $H_{1} \cong H_{2}$. Let $f$ be an isomorphism from $H_{1}$ to $H_{2}$. The amalgamation (the glued graph) of $G_{1}$ and $G_{2}$ at $H_{1}$ and $H_{2}$ with respect to $f$ is the graph $G=\underset{H_{1} \cong_{f} H_{2}}{G_{2}}$ obtained by forming the disjoint union of $G_{1}$ and $G_{2}$ and then identifying $H_{1}$ and $H_{2}$ with respect to $f$. Equivalently, $G=G_{1} \triangleleft \triangleright G_{2}$ is the graph such that

$$
\begin{aligned}
& V(G)=\left(V\left(G_{1}\right)-V\left(H_{1}\right)\right) \cup\left(V\left(G_{2}\right)-V\left(H_{2}\right)\right) \cup\left\{(v, f(v)) \mid v \in V\left(H_{1}\right)\right\} \\
& E(G)=E\left(G_{1}-V\left(H_{1}\right)\right) \cup E\left(G_{2}-V\left(H_{2}\right)\right) \cup\left\{\{u,(v, f(v))\} \mid\{u, v\} \in E\left(G_{1}\right)\right\}
\end{aligned}
$$




$$
\begin{aligned}
& \cup\left\{\{u,(v, f(v))\} \mid\{u, f(v)\} \in E\left(G_{2}\right)\right\} \\
& \cup\left\{\{(u, f(u)),(v, f(v))\} \mid\{u, v\} \in E\left(G_{1}\right) \text { or }\{f(u), f(v)\} \in E\left(G_{2}\right)\right\} .
\end{aligned}
$$

Note that if $\{u, v\} \in E\left(G_{1}\right)$ and $\{f(u), f(v)\} \in E\left(G_{2}\right)$, then $\{u, v\} \in E\left(H_{1}\right)$ and $\{f(u), f(v)\} \in E\left(H_{2}\right)$. As an example, Figure 1 illustrates the amalgamation $G_{1} \triangleleft \triangleright G_{2}$ with $H_{1} \cong_{f} H_{2}$ respect to the isomorphism $f: H_{1} \longrightarrow H_{2}$ defined by $f\left(v_{1}\right)=u_{1}, f\left(v_{2}\right)=u_{2}$, and $f\left(v_{3}\right)=u_{4}$. The amalgamation or the glued graph of two graphs was defined from 2003 by Uiyyasathian [17] for solving the maximal-clique partition problem. In 2006, Promsakon and Uiyyasathian [18] gave an upper bound of the chromatic number of glued graphs in terms of the chromatic numbers of their original graphs. Here, we are interested in finding the domination number of an amalgamation of two cycles at connected subgraphs.

\section{Basic Definitions and Results}

A graph $H$ is a subgraph of a graph $G$ if $V(H) \subseteq V(G)$ and $E(H) \subseteq E(G)$. In this case, we write $H \subseteq G$, and we say that $G$ contains $H$. When $H \subseteq G$ but $H \neq G$, we write $H \subset G$ and call $H$ a proper subgraph of $G$. For a vertex $v$ of a graph $G$, a neighbor of $v$ is a vertex adjacent to $v$ in G. The neighborhood (or open neighborhood) $N(v)$ of $v$ is the set of neighbors of $v$. The closed neighborhood $N[v]$ is defined as $N[v]=N(v) \cup\{v\}$. A vertex $v$ in a graph $G$ is said to dominate itself and each of its neighbors, that is, $v$ dominates the vertices in its closed neighborhood. Therefore, $v$ dominates $1+\operatorname{deg}(v)$ vertices. For a set $S$ of vertices of a graph $G$, the closed neighborhood $N[S]$ is defined as $N[S]=\cup_{v \in S} N[v]$. A set $S$ of vertices of a graph $G$ is said to dominate the vertices in $N[S]$. A set $S$ of vertices of a graph $G$ is a dominating set of $G$ if every vertex of $G$ is dominated by some vertex in $S$, i.e., every vertex in $V(G)-S$ is adjacent to some vertex in $S$. A minimum dominating set in a graph $G$ is a dominating set of minimum cardinality. The cardinality of a minimum dominating set is called the domination number of $G$, and is denoted by $\gamma(G)$. A dominating set of a graph $G$ with minimum cardinality is called a $\gamma-\operatorname{set}$ of $G$.

Since the cardinality of the vertex set of a graph $G$ is finite, the number of dominating sets of $G$ with minimum cardinality is finite too. This gives, for a given graph $G$ of order $n$, the domination number can have a value from the following range: $1 \leq \gamma(G) \leq n$. In particular, $\gamma(G)=1$ if and only if $\Delta=n-1$, where $\Delta$ is the maximum degree of $G$. Let $P_{n}$ denote a path of order $n$ such that $V\left(P_{n}\right)=\left\{v_{1}, v_{2}, \ldots, v_{n}\right\}$ and $E\left(P_{n}\right)=\left\{\left\{v_{i}, v_{i+1}\right\} \mid i=1,2\right.$, $\ldots, n-1\}$. Let $C_{n}$ denote a cycle of order $n(n \geq 3)$ such that $V\left(C_{n}\right)=\left\{v_{1}, v_{2}, \ldots, v_{n}\right\}$ and $E\left(C_{n}\right)=\left\{\left\{v_{i}, v_{i+1}\right\} \mid i=1,2\right.$, $\ldots, n\}$, where + is the addition modulo $n$. It is easy to obtain that $\gamma\left(C_{n}\right)=\gamma\left(P_{n}\right)=\lceil n / 3\rceil$, where $\lceil x\rceil$ is the least integer greater than or equal to $x$.

A graph $G$ is isomorphic to a graph $H$ if there is a bijection $f: V(G) \longrightarrow V(H)$ such that $\{u, v\} \in E(G)$ if and only if $\{f(u), f(v)\} \in E(H)$. If such a function exists, it is called an isomorphism from $G$ to $H$ and written by $G \cong H$. A graph automorphism is simply an isomorphism from a graph to itself. Let $I s o(G, H)$ denote the set of all isomorphisms from a graph $G$ to a graph $H$ and $A u t(G)$ denote the set of all automorphisms on a graph $G$. It is easy to see that if $H$ is a connected subgraph of $C_{n}$, then either $H \cong P_{1}, P_{2}, \ldots, P_{n-1}, P_{n}$ or $C_{n}$. Moreover, Aut $\left(P_{n}, P_{n}\right)=$ $\left\{f_{1}, f_{2}\right\}$ such that $f_{1}\left(v_{i}\right)=v_{i}$ and $f_{2}\left(v_{i}\right)=v_{n-i+1}$ for all $i=1,2, \ldots, n$. Let $H_{1}$ be a connected proper subgraph of $C_{r}$ and $\mathrm{H}_{2}$ a connected proper subgraph of $C_{t}$ such that $V\left(C_{r}\right)=\left\{v_{1}, v_{2}, \ldots, v_{r}\right\}, \quad E\left(C_{r}\right)=\left\{\left\{v_{i}, v_{i+1}\right\} \mid \quad i=1,2, \ldots\right.$, $r\}, V\left(C_{t}\right)=\left\{u_{1}, u_{2}, \ldots, u_{t}\right\}$, and $E\left(C_{t}\right)=\left\{\left\{u_{i}, u_{i+1}\right\} \mid \quad i=\right.$ $1,2, \ldots, t\}$. It follows that if $H_{1} \cong H_{2}$, then $H_{1}$ and $H_{2}$ are paths of order $s$ for some $s \leq \min \{r, t\}$ such that $V\left(H_{1}\right)=$ $\left\{v_{i+1}, v_{i+2}, \ldots, v_{i+s}\right\}$ and $V\left(H_{2}\right)=\left\{u_{j+1}, u_{j+2}, \ldots, u_{j+s}\right\}$, for some $i \in\{1,2, \ldots, r\}$ and $j \in\{1,2, \ldots, t\}$. We thus get Iso $\left(H_{1}, H_{2}\right)=\left\{f_{1}, f_{2}\right\}$ such that $f_{1}\left(v_{i+q}\right)=u_{j+q}$ and $f_{2}\left(v_{i+q}\right)=u_{j+(s-q)+1}$, for all $q=1,2, \ldots, s$. Figures 2 and 3 illustrate $C_{r} \triangleleft \triangleright C_{t}$ and $C_{r} \triangleleft \triangleright C_{t}$, respectively.

$$
H_{1} \cong_{f_{1}} H_{2} \quad H_{1} \cong_{f_{2}} H_{2}
$$

It is easily seen that $\begin{gathered}C_{r} \triangleleft \triangleright C_{t} \cong C_{r} \triangleleft \triangleright C_{t} \text {. Moreover, if } \\ H_{1} \cong \cong_{f_{1}} H_{2} H_{H_{1}} \cong f_{2} H_{2}\end{gathered}$ $H_{1}, H_{1}^{\prime} \subseteq C_{r}$ and $H_{1} \cong H_{1}^{\prime}$, then $C_{r} \triangleleft \triangleright C_{t} \cong C_{r} \triangleleft \triangleright C_{t}$ for all $f \in I s o\left(H_{1}, H_{2}\right)$ and $f^{\prime} \in I s o\left(H_{1}^{\prime}, H_{2}^{\prime}\right)$. This implies the following lemma.

Lemma 1. Let $H_{1} \subseteq C_{r}, H_{1}^{\prime} \subseteq C_{r}, H_{2} \subseteq C_{t}$, and $H_{2}^{\prime} \subseteq C_{t}$ be connected such that $H_{1} \cong H_{1}^{\prime} \cong H_{2} \cong H_{2}^{\prime}$. Then $\gamma\left(\underset{H_{r}}{C_{H_{f}} \triangleleft{ }_{H_{2}}}\right)=$ $\gamma\left(C_{r} \triangleleft \triangleright C_{t}\right)$ for all $f \in I s o\left(H_{1}, H_{2}\right)$ and $f^{\prime} \in I s o\left(H_{1}^{\prime}, H_{2}^{\prime}\right)$. $H_{1}^{\prime} \approx_{f^{\prime}} H_{2}^{\prime}$

The next lemma gives the domination number of $C_{r} \triangleleft \triangleright C_{t}$ for the case $H_{1}=C_{r}$.

$\mathrm{H}_{1} \cong{ }_{f} \mathrm{H}_{2}$

Lemma 2. If $H_{1}=C_{r}$, then $r=t$ and $\gamma\left(\begin{array}{c}\left.C_{r} \triangleleft \triangleright C_{t}\right) \\ H_{1} \cong_{f} H_{2}\end{array}=\lceil r / 3\rceil\right.$.

Proof. Let $H_{1}=C_{r}$ and $G=C_{r} \triangleleft \triangleright C_{t}$. Since $H_{1} \cong H_{2}$, it $\mathrm{H}_{1} \cong_{f} \mathrm{H}_{2}$

follows easily that $r=t$ and $G \cong C_{r}$. We thus get $\gamma(G)=\gamma\left(C_{r}\right)=\lceil r / 3\rceil$.

We now turn to the case $H_{1} \neq C_{r}$. So, $H_{1} \cong H_{2} \cong P_{s}$, for some $s \leq \min \{r, t\}$. Assume, without loss of generality, that $\min \{r, t\}=r$, i.e., $3 \leq r \leq t$. For simplicity of notation, we write $G$ instead of $C_{r} \triangleleft \triangleright C_{t}$. Based on the result of Lemma 1, $H_{1} \cong_{f} H_{2}$

from now on, we can assume that $V\left(H_{1}\right)=\left\{v_{1}, v_{2}, \ldots, v_{s}\right\}$ and $V\left(H_{2}\right)=\left\{u_{1}, u_{2}, \ldots, u_{s}\right\}$, and the isomorphism $f: H_{1} \longrightarrow H_{2}$ is defined by $f\left(v_{i}\right)=u_{i}$, for all $i=1,2, \ldots, s$. So, $\left(v_{1}, u_{1}\right),\left(v_{2}, u_{2}\right), \ldots,\left(v_{s}, u_{s}\right) \in V(G)$. Now, we consider $\gamma(G)$ with $H_{1} \cong P_{1}$.

Lemma 3. If $H_{1} \cong P_{1}$, then $\gamma(G)=\lceil(r-3) / 3\rceil+\lceil(t-3) / 3\rceil+1$. 

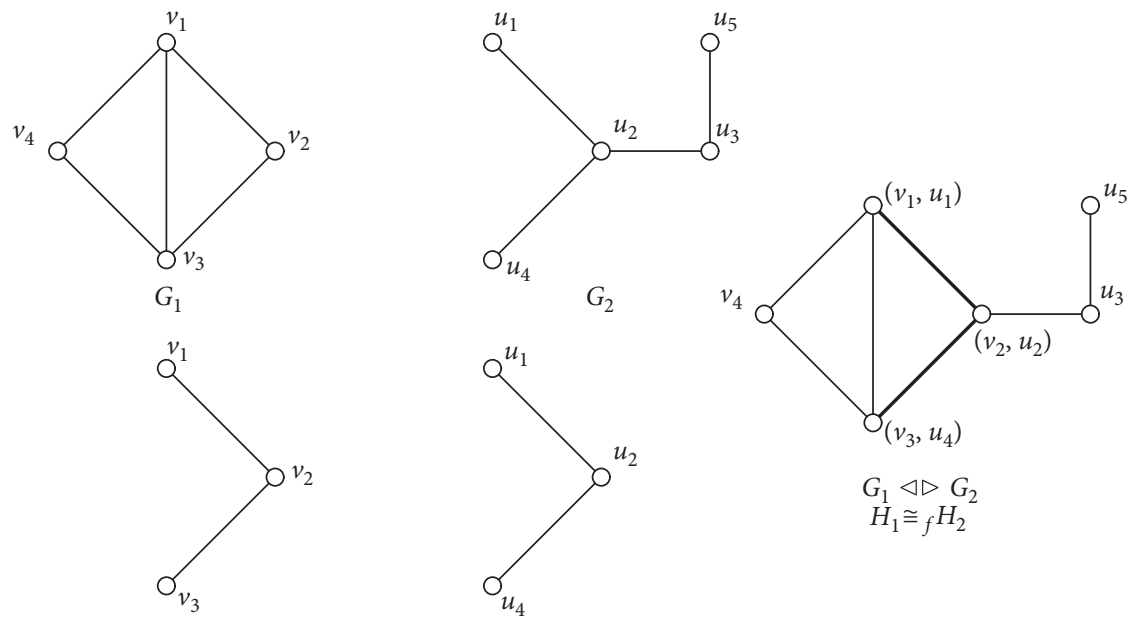

$H_{1}$

$\mathrm{H}_{2}$

Figure 1: An amalgamation of $G_{1}$ and $G_{2}$ at $H_{1}$ and $H_{2}$ with respect to $f$.

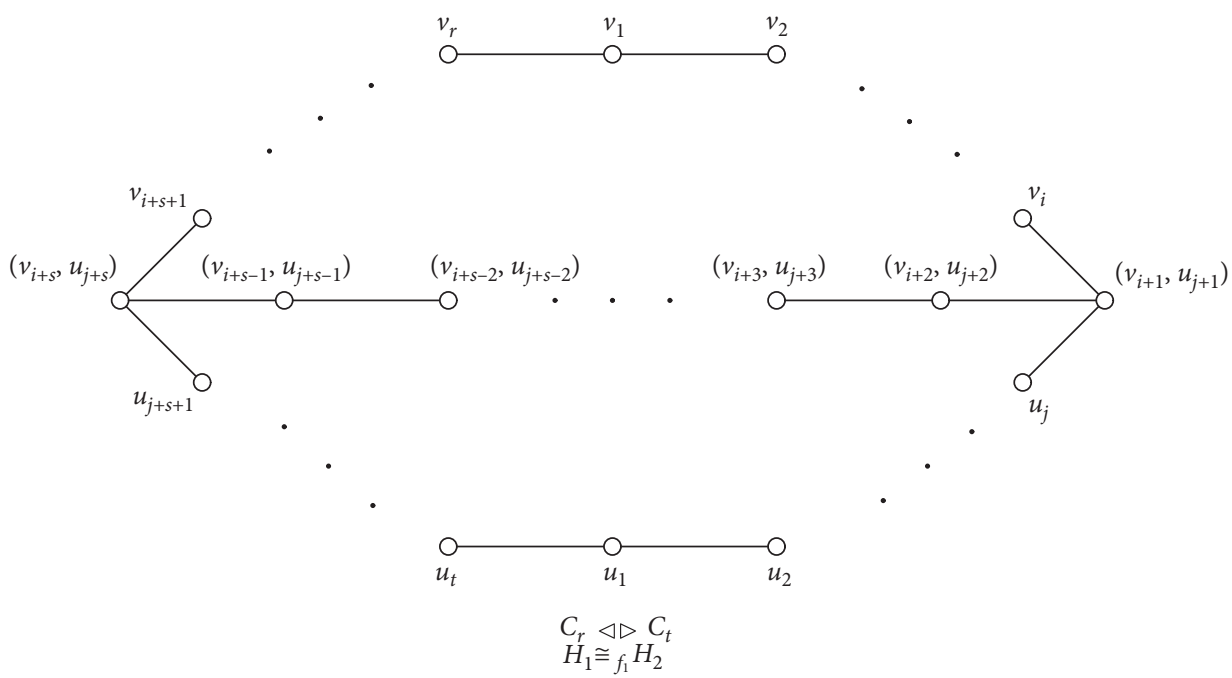

Figure 2: The amalgamation of $C_{r}$ and $C_{t}$ at connected proper subgraphs $H_{1}$ and $H_{2}$ with respect to $f_{1}$.

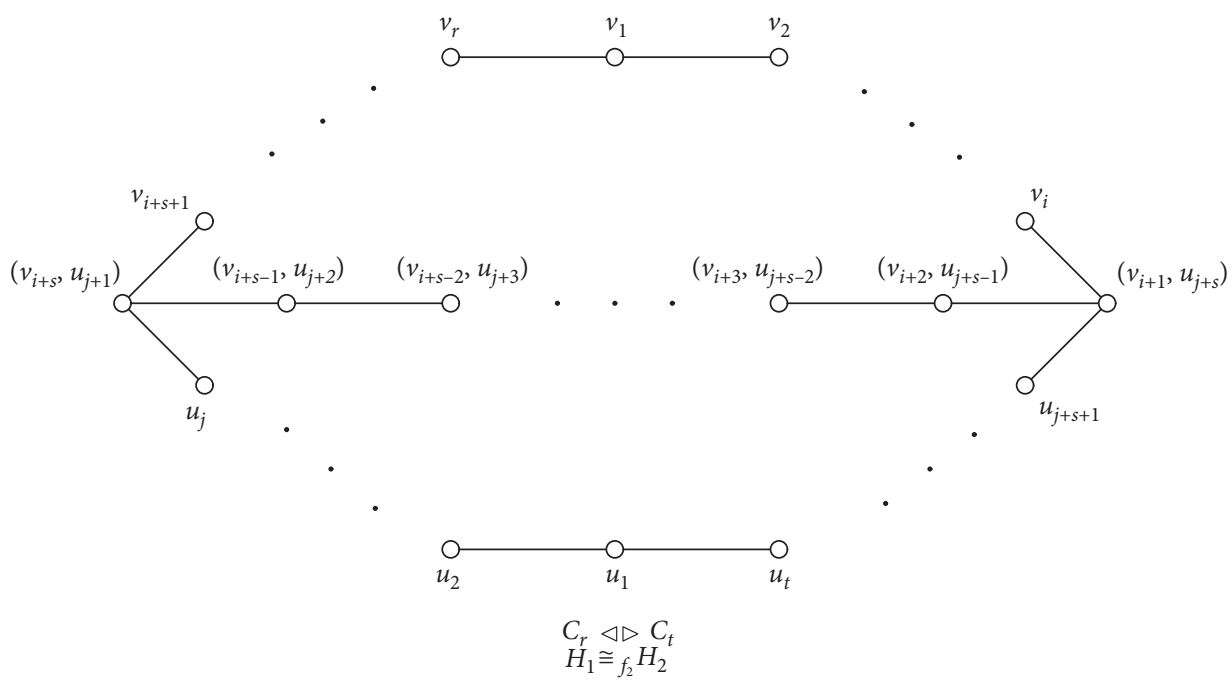

Figure 3: The amalgamation of $C_{r}$ and $C_{t}$ at connected proper subgraphs $H_{1}$ and $H_{2}$ with respect to $f_{2}$. 
Proof. Suppose that $H_{1} \cong P_{1}$. Then, $\left(v_{1}, u_{1}\right) \in V(G)$ (see Figure 4(a)).

Let $S=\left\{\left(v_{1}, u_{1}\right)\right\} \cup\left\{v_{i} \mid i \equiv 1 \quad(\bmod 3), 3 \leq i \leq r-1\right\} \cup$ $\left\{u_{i} \mid i \equiv 1 \quad(\bmod 3), 3 \leq i \leq t-1\right\}$. We check at once that $S$ is a dominating set of $G$ and $|S|=\lceil(r-3) / 3\rceil+\lceil(t-3) / 3\rceil+1$. This gives $\gamma(G) \leq\lceil(r-3) / 3\rceil+\lceil(t-3) / 3\rceil+1$. Let $S^{\prime}$ be a $\gamma-$ set of $G$. Thus, $\left(v_{1}, u_{1}\right) \in S^{\prime}$ since otherwise $S^{\prime}$ is not a $\gamma$ - set of $G$. In order to dominate the vertices in $G-N\left[\left\{\left(v_{1}, u_{1}\right)\right\}\right]$, then $S-\left\{\left(v_{1}, u_{1}\right)\right\}$ must contain at least $\lceil(r-3) / 3\rceil+\lceil(t-3) / 3\rceil$ vertices. This gives $\gamma(G)=\left|S^{\prime}\right| \geq\lceil(r-3) / 3\rceil+\lceil(t-3) / 3\rceil+1$. It follows that $\gamma(G)=\lceil(r-3) / 3\rceil+\lceil(t-3) / 3\rceil+1$.

Now, we consider $\gamma(G)$ with $H_{1} \cong P_{r}$ and define sets $S$ and $S^{\prime}$ as follows:

$$
\begin{aligned}
& S=\left\{\left(v_{i}, u_{i}\right) \mid i \equiv 1 \quad(\bmod 3), 1 \leq i \leq r\right\} \cup\left\{u_{i} \mid i \equiv 2\right. \\
& (\bmod 3), r+1 \leq i \leq t\}, \\
& S^{\prime}=\left\{\left(v_{i}, u_{i}\right) \mid i \equiv 1 \quad(\bmod 3), 1 \leq i \leq r\right\} \cup\left\{u_{i} \mid i \equiv 1\right. \\
& (\bmod 3), r+1 \leq i \leq t\} .
\end{aligned}
$$

Note that $|S|=\lceil r / 3\rceil+\lceil(t-r-1) / 3\rceil$ and $\left|S^{\prime}\right|=\lceil t / 3\rceil$.

Lemma 4. Let $H_{1} \cong P_{r}$. Then,

$\gamma(G)= \begin{cases}\lceil r / 3\rceil+\lceil(t-r-1) / 3\rceil & \text { if } \quad r \equiv 0(\bmod 3) \text { and } t \equiv 1(\bmod 3) \\ \lceil t / 3\rceil & \text { otherwise. }\end{cases}$

Proof. Suppose that $H_{1} \cong P_{r}$. Then, $\left(v_{1}, u_{1}\right), \ldots$, $\left(v_{r}, u_{r}\right) \in V(G)$ (see Figure 5(a)).

If $r=t$, then $G$ is the graph obtained from $C_{t}$ by joining $v_{1}$ and $v_{t}$ with a new edge. It follows easily that $\gamma(G)=\gamma\left(C_{t}\right)=\lceil t / 3\rceil$. For $r \neq t$, we consider two cases.

Case $1 . r \equiv 0 \quad(\bmod 3)$ and $t \equiv 1 \quad(\bmod 3)$. We check at once that the set $S$ defined above is a dominating set of $G$. Thus, $\gamma(G) \leq\lceil r / 3\rceil+\lceil(t-r-1) / 3\rceil$. We now prove that $\gamma(G) \geq\lceil r / 3\rceil+\lceil(t-r-1) / 3\rceil$. Let $S_{1}$ be a $\gamma-$ set of $G$. In order to dominate $\left(v_{1}, u_{1}\right), S_{1}$ must contain at least one vertex of $\left\{\left(v_{1}, u_{1}\right),\left(v_{2}, u_{2}\right),\left(v_{r}, u_{r}\right), u_{t}\right\}$. There are two possible cases: either $\left(v_{1}, u_{1}\right) \in S_{1}$ or $\left(v_{r}, u_{r}\right) \in S_{1}$ since otherwise $S_{1}$ is not a $\gamma-$ set of $G$. We give the proof only for the case $\left(v_{1}, u_{1}\right) \in S_{1}$; the proof of the case $\left(v_{r}, u_{r}\right) \in S_{1}$ is similar. Consider the vertices of $G-N\left[\left\{\left(v_{1}, u_{1}\right)\right\}\right] \cong P_{r-3} \cup P_{t-r-1}$. In order to dominate $\left(v_{3}, u_{3}\right), \ldots,\left(v_{r-1}, u_{r-1}\right)$ and $u_{r+1}, \ldots, u_{t-1}$, there are at least $\lceil(r-3) / 3\rceil+\lceil(t-r-1) / 3\rceil$ vertices of $\left\{\left(v_{2}, u_{2}\right), \ldots,\left(v_{r-1}, u_{r-1}\right)\right\} \cup\left\{u_{r+1}, \ldots, u_{t}\right\}$ in $S_{1}-\left\{\left(v_{1}\right.\right.$, $\left.\left.u_{1}\right)\right\}$. This gives $\left|S_{1}\right| \geq 1+\lceil(r-3) / 3\rceil+\lceil(t-r-1) / 3\rceil$, so $\gamma(G) \geq\lceil r / 3\rceil+\lceil(t-r-1) / 3\rceil$. Hence, $\gamma(G)=\lceil r / 3\rceil$ $+\lceil(t-r-1) / 3\rceil$.

Case 2. $r \equiv 0 \quad(\bmod 3)$ or $t \equiv 1 \quad(\bmod 3)$. We see at once that the set $S^{\prime}$ defined above is a dominating set of $G$. Thus, $\gamma(G) \leq\lceil t / 3\rceil$. We now prove that $\gamma(G) \geq\lceil t / 3\rceil$. Let $S_{2}$ be a $\gamma$ - set of $G$. We consider two subcases.

Case 2.1. $r \equiv 0 \quad(\bmod 3)$ and $t \equiv 0 \quad(\bmod 3)$ and $r \equiv 1 \quad(\bmod 3)$ and $t \equiv a \quad(\bmod 3)$, for some $a \in\{0,1,2\}$, or $r \equiv 2(\bmod 3)$ and $t \equiv a \quad(\bmod 3)$, for some $a \in\{0,2\}$.
Case 2.1.1 $\left(v_{1}, u_{1}\right) \in S_{2}$ or $\left(v_{r}, u_{r}\right) \in S_{2}$. If $\left(v_{1}\right.$, $\left.u_{1}\right) \in S_{2}$ and $t>r+1$, then, in order to dominate $\left(v_{3}, u_{3}\right), \ldots,\left(v_{r-1}, u_{r-1}\right)$ and $u_{r+2}, \ldots, u_{t-1}$, there are at least $\lceil(r-3) / 3\rceil+\lceil(t-r-2) / 3\rceil$ vertices of $\left\{\left(v_{2}, u_{2}\right), \ldots,\left(v_{r}, u_{r}\right)\right\} \cup\left\{u_{r+1}, \ldots, u_{t}\right\}$ in $S_{2}-\left\{\left(v_{1}, u_{1}\right.\right.$ )$\}$. It follows that $\left|S_{2}\right| \geq 1+\lceil(r-3) / 3\rceil+\lceil(t-r-2)$ $/ 3\rceil=\lceil t / 3\rceil$. If $\left(v_{1}, u_{1}\right) \in S_{2}$ and $t=r+1$, then $\left\{u_{r+2}, \ldots u_{t-1}\right\}=\varnothing$. So, in order to dominate the vertices in $G-N\left[\left\{\left(v_{1}, u_{1}\right)\right\}\right]$, there are at least $\lceil(r-3) / 3\rceil$ vertices of $\left\{\left(v_{2}, u_{2}\right), \ldots,\left(v_{r}, u_{r}\right)\right\}$ in $S_{2}-\left\{\left(v_{1}, u_{1}\right)\right\}$. This gives $\left|S_{2}\right| \geq 1+\lceil(r-3) / 3\rceil=\lceil t$ 13†. Similarly, if $\left(v_{r}, u_{r}\right) \in S_{2}$, then $\left|S_{2}\right| \geq\lceil t / 3\rceil$. Thus, $\gamma(G) \geq\lceil t / 3\rceil$.

Case 2.1.2. $\left(v_{1}, u_{1}\right) \notin S_{2}$ and $\left(v_{r}, u_{r}\right) \notin S_{2}$. Clearly, $\gamma(G)=\left|S_{2}\right| \geq \gamma\left(C_{t}\right)=\lceil t / 3\rceil$.

Case 2.2. $r \equiv 0 \quad(\bmod 3)$ and $t \equiv 2(\bmod 3)$ or $r \equiv 2 \quad(\bmod 3)$ and $t \equiv 1 \quad(\bmod 3)$.

Case 2.2.1. either $\left(v_{1}, u_{1}\right) \in S_{2}$ or $\left(v_{r}, u_{r}\right) \in S_{2}$. If $\left(v_{1}, u_{1}\right) \in S_{2}$, then, in order to dominate $\left(v_{3}, u_{3}\right), \ldots,\left(v_{r}, u_{r}\right), u_{r+1}, \ldots, u_{t-1}$ in $G$, there are at least $\lceil(t-3) / 3\rceil$ vertices of $\left\{\left(v_{2}, u_{2}\right), \ldots,\left(v_{r}\right.\right.$, $\left.\left.u_{r}\right), u_{r+1}, \ldots, u_{t}\right\}$, in $S_{2}-\left\{\left(v_{1}, u_{1}\right)\right\}$. It follows that $\left|S_{2}\right| \geq 1+\lceil(t-3) / 3\rceil=\lceil t / 3\rceil$. Similarly, if $\left(v_{r}, u_{r}\right) \in$ $S_{2}$, then $\left|S_{2}\right| \geq\lceil t / 3\rceil$. Thus, $\gamma(G) \geq\lceil t / 3\rceil$.

Case 2.2.2. $\left(v_{1}, u_{1}\right) \in S_{2}$ and $\left(v_{r}, u_{r}\right) \in S_{2}$. If $r>3$ and $t>r+1$, then, in order to dominate $\left(v_{3}, u_{3}\right), \ldots$, $\left(v_{r-2}, u_{r-2}\right)$ and $u_{r+2}, \ldots, u_{t-1}$, there are at least $\lceil(r-4) / 3\rceil+\lceil(t-r-2) / 3\rceil$ vertices of $\left\{\left(v_{2}, u_{2}\right), \ldots\right.$, $\left.\left(v_{r-1}, u_{r-1}\right)\right\} \cup\left\{u_{r+1}, \ldots, u_{t}\right\} \quad$ in $S_{2}$. Hence, $\left|S_{2}\right| \geq 2+\lceil(r-4) / 3\rceil+\lceil(t-r-2) / 3\rceil=\lceil t / 3\rceil$. If $r=$ 3 and $t>r+1$, then there are at least $\lceil(t-r-2) / 3\rceil$ vertices of $\left\{u_{r+1}, \ldots, u_{t}\right\}$ in $S_{2}$. The result is $\left|S_{2}\right| \geq 2+\lceil(t-r-2) / 3\rceil=\lceil t / 3\rceil$. If $r>3$ and $t=r$ +1 , then there are at least $\lceil(r-4) / 3\rceil$ vertices of $\left\{\left(v_{2}, u_{2}\right), \ldots,\left(v_{r-1}, u_{r-1}\right)\right\}$. We thus get $\left|S_{2}\right| \geq$ $2+\lceil(r-4) / 3\rceil=\lceil t / 3\rceil$. Clearly, if $r=3$ and $t=r+1$, then $t=4$ and $\left|S_{2}\right| \geq 2=\lceil t / 3\rceil$. Thus, $\gamma(G) \geq\lceil t / 3\rceil$.

Case 2.2.3. $\left(v_{1}, u_{1}\right) \notin S_{2}$ and $\left(v_{r}, u_{r}\right) \notin S_{2}$. Clearly, $\gamma(G)=\left|S_{2}\right| \geq \gamma\left(C_{t}\right)=\lceil t / 3\rceil$.

Lemma 5. If $H_{1} \cong P_{2}$, then

$\gamma(G)= \begin{cases}\left\lceil\frac{r}{3}\right\rceil+\left\lceil\frac{t-3}{3}\right\rceil & \text { if } r \equiv 0 \quad(\bmod 3) \text { and } t \equiv 0 \quad(\bmod 3), \\ \left\lceil\frac{t+r-2}{3}\right\rceil & \text { otherwise. }\end{cases}$

Proof. Suppose that $H_{1} \cong P_{2}$. It is easy to check that $G=$ $C_{r} \triangleleft \triangleright C_{t} \cong C_{r} \triangleleft \triangleright C_{t^{\prime}}$ where $H_{1}^{\prime} \cong P_{r}$ and $t^{\prime}=t+r-2$ (see $H_{1} \cong_{f} H_{2} \quad H_{1}^{\prime} \cong_{f^{\prime}} H_{2}^{\prime}$

Figures 4(b) and 5(a)). By Lemma 4, the result holds.

Next, we will give the domination number of $G$ such that $H_{1} \cong P_{3}(r>3)$. We define three sets $S_{0}, S_{1}$, and $S_{2}$ as follows: 


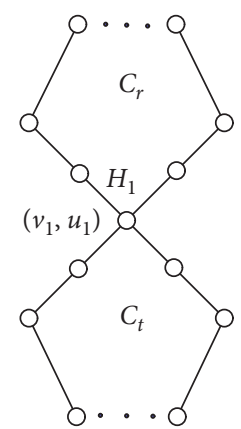

(a)

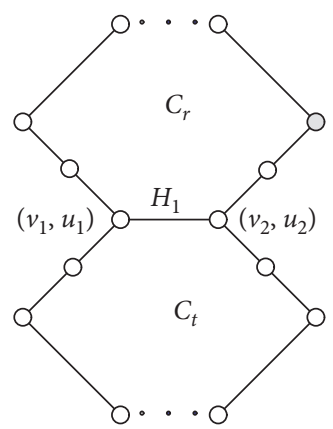

(b)

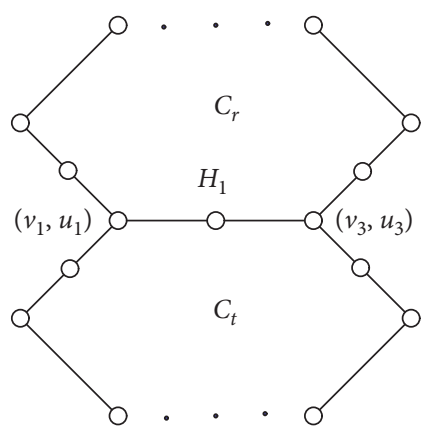

(c)

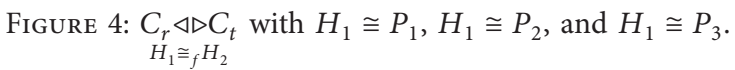

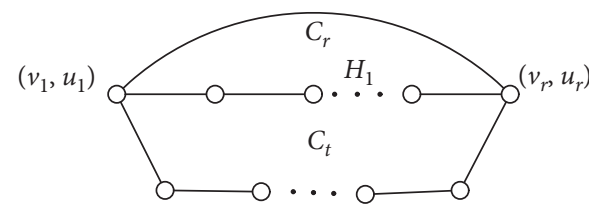

(a)

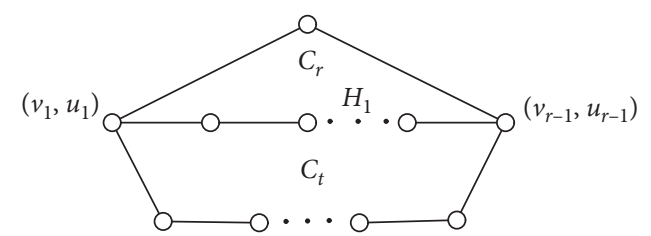

(b)

FIgURE 5: $\begin{array}{r}C_{r} \triangleleft \triangleright C_{t} \text { with } H_{1} \cong P_{r} \text { and } H_{1} \cong P_{r-1} \text {. } \\ H_{1} \cong_{f} H_{2}\end{array}$

$$
\begin{aligned}
& S_{0}=\left\{\left(v_{1}, u_{1}\right)\right\} \cup\left\{u_{i} \mid i \equiv 1 \quad(\bmod 3), 4 \leq i \leq t\right\} \cup\left\{v_{i} \mid i \equiv 2\right. \\
& (\bmod 3), 4 \leq i \leq r\} \text {. } \\
& S_{1}=\left\{\left(v_{1}, u_{1}\right)\right\} \cup\left\{u_{i} \mid i \equiv 2 \quad(\bmod 3), 4 \leq i \leq t\right\} \cup\left\{v_{i} \mid i \equiv 1\right. \\
& (\bmod 3), 4 \leq i \leq r\} \text {. } \\
& S_{2}=\left\{\left(v_{1}, u_{1}\right)\right\} \cup\left\{u_{i} \mid i \equiv 0 \quad(\bmod 3), 4 \leq i \leq t\right\} \cup\left\{v_{i} \mid i \equiv 0\right. \\
& (\bmod 3), 4 \leq i \leq r\} \text {. }
\end{aligned}
$$

Note that if $t \equiv j \quad(\bmod 3)$, for some $j \in\{0,1,2\}$, then $\left|S_{j}\right|=\lceil(r+t-7) / 3\rceil+1$.

Lemma 6. If $H_{1} \cong P_{3}$ and $r>3$, then $\gamma(G)=$ $\lceil(r+t-7) / 3\rceil+1$.

Proof. Suppose that $H_{1} \cong P_{3}$. Then, $\left(v_{1}, u_{1}\right),\left(v_{2}, u_{2}\right),\left(v_{3}, u_{3}\right) \in V(G)$ (see Figure $\left.4(\mathrm{c})\right)$. Note that if $t \equiv j \quad(\bmod 3)$, for some $j \in\{0,1,2\}$, then the set $S_{j}$ defined above is a dominating set of $G$, so $\gamma(G) \leq\lceil(r+$ $t-7) / 3\rceil+1$. We now prove that $\gamma(G) \geq\lceil(r+t-7) / 3\rceil+1$. Let $S$ be a $\gamma$ - set of $G$. If $r=4$, then, in order to dominate vertices $\left(v_{1}, u_{1}\right),\left(v_{2}, u_{2}\right),\left(v_{3}, u_{3}\right), u_{4}, \ldots, u_{t}$, there are at least $\lceil t / 3\rceil$ vertices of $\left\{\left(v_{1}, u_{1}\right),\left(v_{2}, u_{2}\right),\left(v_{3}, u_{3}\right), u_{4}, \ldots, u_{t}\right\} \cup\left\{v_{4}\right\}$ in $S$. Thus, $\gamma(G)=|S| \geq\lceil t / 3\rceil=\lceil(4+t-7) / 3\rceil+1$. For $r \geq 5$, we consider two cases.

Case 1. $\left(v_{1}, u_{1}\right) \in S$. In this case, we consider two subcases.

Case 1.1. $\left(v_{3}, u_{3}\right) \in S$. Consider the vertices in $G-N\left[\left\{\left(v_{1}, u_{1}\right),\left(v_{3}, u_{3}\right)\right\}\right] \cong P_{r-5} \cup P_{t-5}$. We see that there are at least $\lceil(r-5) / 3\rceil+\lceil(t-5) / 3\rceil$ vertices in $S-\left\{\left(v_{1}, u_{1}\right),\left(v_{3}, u_{3}\right)\right\}$. This gives $|S| \geq\lceil(r-5) / 3\rceil$ $+\lceil(t-5) / 3\rceil+2 \geq\lceil(r+t-7) / 3\rceil+1$.
Case 1.2. $\left(v_{3}, u_{3}\right) \notin S$. Consider the vertices in $G-N\left[\left\{\left(v_{1}, u_{1}\right)\right\}\right] \cong P_{r+t-7}$. We see at once that there are at least $\lceil(r+t-7) / 3\rceil$ vertices in $S-\left\{\left(v_{1}, u_{1}\right)\right\}$. It follows that $|S| \geq\lceil(r+t-7) / 3\rceil+1$.

Case 2. $\left(v_{1}, u_{1}\right) \notin S$. In order to dominate $\left(v_{1}, u_{1}\right)$, one vertex of $\left\{\left(v_{2}, u_{2}\right), v_{r}, u_{t}\right\}$ must be in $S$.

Case 2.1. $\left(v_{2}, u_{2}\right) \in S$. Consider the vertices in $G-N\left[\left\{\left(v_{2}, u_{2}\right)\right\}\right]$. In order to dominate $v_{4}, \ldots, v_{r}$ and $u_{5}, \ldots, u_{t}$, there are at least $\lceil(r-3) / 3\rceil+\lceil(t-4) / 3\rceil$ vertices in $S-\left\{\left(v_{2}, u_{2}\right)\right\}$. It follows that $|S| \geq$ $\lceil(r-3) / 3\rceil+\lceil(t-4) / 3\rceil+1 \geq\lceil(r+t-7) / 3\rceil+1$.

Case 2.2. $v_{r} \in S$. If $\left(v_{2}, u_{2}\right) \in S$, then this case is the same as Case 2.1. If $\left(v_{2}, u_{2}\right) \notin S$, then, in order to dominate $u_{t}, \ldots, u_{4},\left(v_{3}, u_{3}\right), v_{4}, \ldots, v_{r-2} \quad$ in $G-N\left[\left\{v_{r}\right\}\right], S-\left\{v_{r}\right\}$ contains at least $\lceil(t+r-7) / 3\rceil$ vertices. This gives $|S| \geq\lceil(r+t-7) / 3\rceil+1$.

Case 2.3. $u_{t} \in S$. By the same argument as in Case 2.2, we also get $|S| \geq\lceil(r+t-7) / 3\rceil+1$.

Lemma 7. If $H_{1} \cong P_{r-1}$, then $\gamma(G)=\lceil(t-3) / 3\rceil+1$.

Proof. Suppose that $H_{1} \cong P_{r-1}$. It is easy to check that $G=C_{r} \triangleleft \triangleright C_{t} \cong C_{r} \triangleleft \triangleright C_{t^{\prime}}$, where $H_{1}^{\prime} \cong P_{3}$ and $t^{\prime}=t-r+4$ $H_{1} \cong_{f} H_{2} \quad H_{1}^{\prime} \approx_{f^{\prime}} H_{2}^{\prime}$

(see Figures 4(c) and 5(b)). By Lemma 6, the result holds.

By Lemma 3-7, we know the domination number of $G$, for all $r$ such that $3 \leq r \leq 5$. We also know the domination number of $G$, for all $H_{1}$ such that $H_{1} \cong P_{1}, P_{2}, P_{3}, P_{r-1}, P_{r}$. We now consider the case $r \geq 6$ and $H_{1} \cong P_{4}, P_{5}, \ldots, P_{r-2}$. 
For $n, k, m \in \mathbb{N}-\{1,2\}$, let us denote by $C(m, k, n)$ a graph with $V(C(m, k, n))=\{1, x\} \cup\left\{2_{m}, 3_{m}, \ldots, m_{m}\right\} \cup$ $\left\{2_{k}, 3_{k}, \ldots, k_{k}\right\} \cup\left\{2_{n}, 3_{n}, \ldots, n_{n}\right\} \quad$ and $E(C(m, k, n))$ $=\cup_{q \in\{m, k, n\}}\left\{\left\{1, \quad 2_{q}\right\},\left\{2_{q}, 3_{q}\right\}, \ldots,\left\{(q-1)_{q}, q_{q}\right\},\left\{q_{q}, x\right\}\right\}$. Figure 6 illustrates $C(m, k, n)$.

Lemma 8. If $H_{1} \cong P_{s}$, for some $s \in\{4,5, \ldots, r-2\}$ and $r \geq 6$, then $G \cong C(m, k, n)$ where $r=m+k, s=k+1$, and $t=n+k$.

Proof. Suppose that $H_{1} \cong P_{s}$, for some $s \in\{4,5, \ldots, r-2\}$ and $r \geq 6$. Let $r=m+k, s=k+1$, and $t=n+k$. Define $f: V(G) \longrightarrow V(C(m, k, n))$ by

$f(g)= \begin{cases}1 & \text { if } g=\left(v_{1}, u_{1}\right), \\ i_{k} & \text { if } g=\left(v_{i}, u_{i}\right) \text { and } 2 \leq i \leq s-1, \\ (m-i+1)_{m} & \text { if } g=v_{s+i} \text { and } 1 \leq i \leq r-s, \\ (n-i+1)_{n} & \text { if } g=u_{s+i} \text { and } 1 \leq i \leq t-s, \\ x & \text { if } g=\left(v_{s}, u_{s}\right) .\end{cases}$

It is easy to check that $f$ is an isomorphism. Then, $G \cong C(m, k, n)$.

\section{Domination Numbers of Amalgamations of Cycles at Connected Subgraphs}

In this section, we calculate the domination number $\gamma(C(m, k, n))$. Then, by Lemma 8 , we thus get the domination number $\gamma(G)$ for the case $r \geq 6$ and $H_{1} \cong P_{4}, P_{5}, \ldots, P_{r-2}$. The following two lemmas provide upper bounds of $\gamma(C(m, k, n))$.

Lemma 9. Let $m, k, n \in \mathbb{N}-\{1,2\}$ and $S=\{1, x\} \cup$ $\cup_{q \in\{m, k, n\}}\left\{a_{q} \mid a \equiv 1 \quad(\bmod 3), 3 \leq a \leq q-1\right\}$. Then, $S$ is $a$ dominating set of $C(m, k, n)$ and $|S|=2+\lceil(m-3) / 3\rceil$ $+\lceil(k-3) / 3\rceil+\lceil(n-3) / 3\rceil$.

Proof. Let $v \in V(C(m, k, n))-S$. If $v=2_{q}$, for some $q \in\{m, k, n\}$, then there is $1 \in S$ such that $\{1, v\} \in E(C(m, k, n))$. If $v=q_{q}$, for some $q \in\{m, k, n\}$, then there is $x \in S$ such that $\{x, v\} \in E(C(m, k, n))$. If $v=u_{q}$, for some $q \in\{m, k, n\}$, such that $u \equiv 0(\bmod 3)$ and $u \notin\{m, k, n\}$, then $u+1 \equiv 1 \quad(\bmod 3)$ and thus $(u+1)_{q} \in S$. It follows that there is $(u+1)_{q} \in S$ such that $\left\{v,(u+1)_{q}\right\} \in E(C(m, k, n))$. If $v=u_{q}$, for some $q \in\{m, k, n\}$, such that $u \equiv 2 \quad(\bmod 3)$ and $u \notin\{2, m, k, n\}$, then $u-1 \equiv 1 \quad(\bmod 3)$ and thus $(u-1)_{q} \in S$. Hence, there is $(u-1)_{q} \in S$ such that $\left\{v,(u-1)_{q}\right\} \in E(C(m, k, n))$. Therefore, $S$ is a dominating set of $C(m, k, n)$. It is easily seen that $|S|=2+\lceil(m-3) / 3\rceil+\lceil(k-3) / 3\rceil+\lceil(n-3) / 3\rceil$.

Lemma 10. Let $n, k, m \in \mathbb{N}-\{1,2\}$ and $S=\{1\} \cup$ $\cup_{q \in\{m, k, n\}}\left\{a_{q} \mid a \equiv 1 \quad(\bmod 3), 3 \leq a \leq q\right\}$. If $m, k, n$ $\equiv 0 \quad(\bmod 3)$ and $z \equiv 1 \quad(\bmod 3)$, for some $z \in\{m, k, n\}$, then $S$ is a dominating set of $C(m, k, n)$ and $|S|=1+\lceil(m-2) / 3\rceil+\lceil(k-2) / 3\rceil+\lceil(n-2) / 3\rceil$.
Proof. Suppose that $m, k, n \equiv 0 \quad(\bmod 3)$ and $z \equiv 1 \quad(\bmod 3)$, for some $z \in\{m, k, n\}$. Without loss of generality, we can assume that $m \equiv 1 \quad(\bmod 3)$. We will prove that $S$ is a dominating set of $C(m, k, n)$. There are four cases for $k$ and $n$. We give the proof only for the case $k \equiv 1 \quad(\bmod 3)$ and $n \equiv 2 \quad(\bmod 3)$; the proofs of the other cases are similar. Let $v \in V(C(m, k, n))-S$. If $v=2_{q}$, for some $q \in\{m, k, n\}$, then there is $1 \in S$ such that $\{1, v\} \in E(C(m, k, n))$. If $v=u_{q}$, for some $q \in\{m, k, n\}$ such that $u \equiv 0 \quad(\bmod 3)$, then $u+1 \equiv 1 \quad(\bmod 3)$ and so $(u+1)_{q} \in S$. Thus, there is $(u+1)_{q} \in S$ such that $\left\{v,(u+1)_{q}\right\} \in E(C(m, k, n))$. If $v=u_{q}$, for some $q \in\{m, k, n\}$ such that $u \equiv 2(\bmod 3)$ and $u \neq 2$, then $u-1 \equiv 1 \quad(\bmod 3)$, and thus, $(u-1)_{q} \in S$. Consequently, there is $(u-1)_{q} \in S$ such that $\left\{v,(u-1)_{q}\right\} \in E(C(m, k, n))$. If $v=x$, then there is $m_{m} \in S$ such that $\left\{m_{m}, v\right\} \in E(C(m, k, n))$. Therefore, $S$ is a dominating set of $C(m, k, n)$. It is easy to check that $|S|=1+$ $\lceil(m-3) / 3\rceil+\lceil(k-3) / 3\rceil+\lceil(n-3) / 3\rceil$.

The following lemmas about $\gamma$-set will be used in Theorem 1 to determine lower bounds of $\gamma(C(m, k, n))$.

Lemma 11. Let $n, k, m \in \mathbb{N}-\{1,2\}$ such that $y \equiv 0 \quad(\bmod 3)$, for all $y \in\{m, k, n\}$ and $z \equiv 1 \quad(\bmod 3)$, for some $z \in\{m, k, n\}$, and let $S$ be a $\gamma$-set of $C(m, k, n)$. Then, the following hold:

(1) $|S \cap\{1, x\}| \leq 1$.

(2) If $1 \in S$, then $q_{q} \in S$ for some $q \in\{m, k, n\}$ such that $q \equiv 1 \quad(\bmod 3)$.

(3) If $x \in S$, then $2_{q} \in S$ for some $q \in\{m, k, n\}$ such that $q \equiv 1 \quad(\bmod 3)$.

Proof. Without loss of generality, we can assume that $m \equiv 1 \quad(\bmod 3)$.

(1) There are four cases for $k$ and $n$. We give the proof only for the case $k \equiv 1 \quad(\bmod 3)$ and $n \equiv 2 \quad(\bmod 3)$; the proofs of the other cases are similar. On the contrary, suppose that $|S \cap\{1, x\}|=2$. This gives $1, x \in S$, and thus, every vertex in $N[\{1, x\}]=\left\{1,2_{m}, 2_{k}, 2_{n}, x, m_{m}, k_{k}, n_{n}\right\}$ is dominated by $\quad S$. Obviously, $C(m, k, n)-N[\{1, x\}]$ $\cong P_{m-3} \cup P_{k-3} \cup P_{k-3}$ (see Figure 7 ).

In order to dominate the vertices in $C(m, k, n)-N[\{1, x\}]$, then $S-\{1, x\}$ must contain at least $\lceil(m-3) / 3\rceil+\lceil(k-3) / 3\rceil+\lceil(n-3) / 3\rceil$ vertices. This gives $|S| \geq 2+\lceil(m-3) / 3\rceil+\lceil(k-3) / 3\rceil+\lceil(n-3)$ $13\rceil=2+\lceil(m-2) / 3\rceil+\lceil(k-2) / 3\rceil+\lceil(n-2) / 3\rceil$. From Lemma 10 , we know that $C(m, k, n)$ contains a dominating set of order $1+\lceil(m-2) / 3\rceil+$ $\lceil(k-2) / 3\rceil+\lceil(n-2) / 3\rceil$. This contradicts the fact that $S$ is a $\gamma-$ set of $C(m, k, n)$.

(2) Let $1 \in S$. By (1), $x \notin S$. Suppose, by contrary, $q_{q} \notin S$, for all $q \in\{m, k, n\}$ such that $q \equiv 1 \quad(\bmod 3)$. In order to dominate $x$, then there exists $z_{z} \in S-\{1\}$, for some $z \in\{m, k, n\}$ such that $z \neq m$ and $z \equiv 2(\bmod 3)$. There are two cases for $z$. We give 

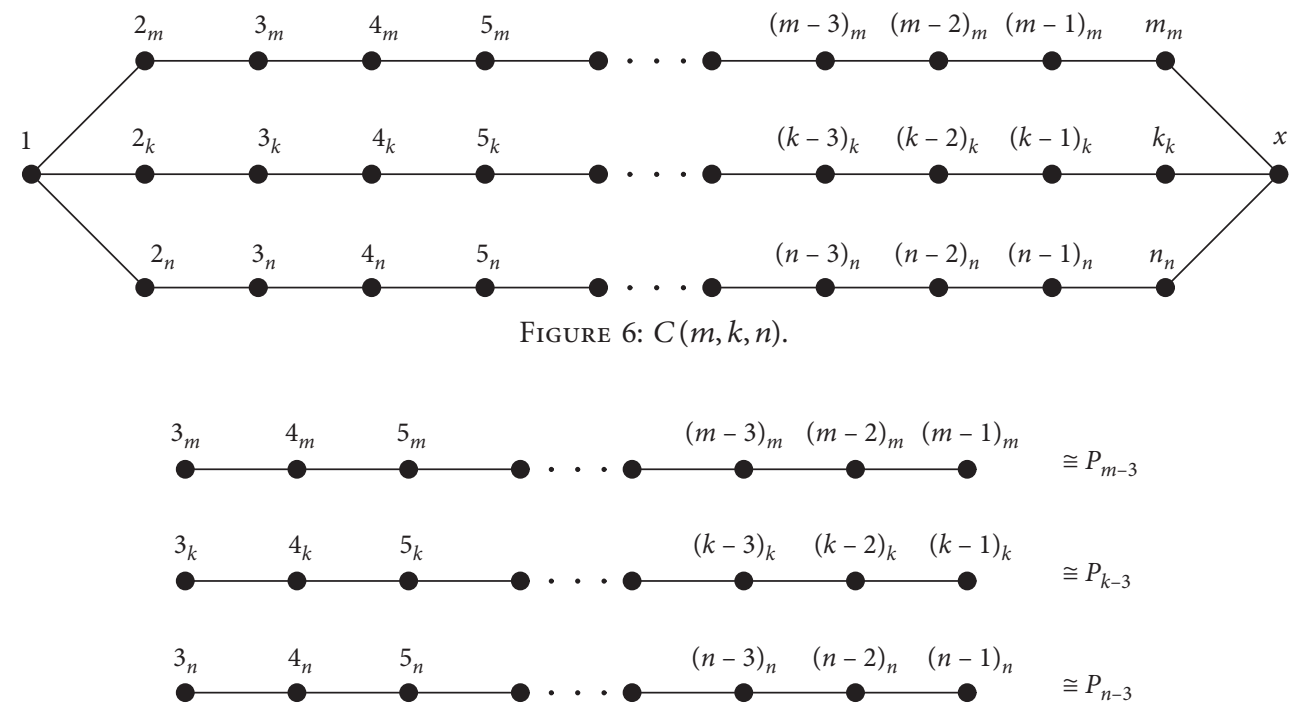

Figure 7: $C(m, k, n)-N[\{1, x\}]$.

the proof only for the case $z=n$; the proof of the case $z=k$ is similar. Since $1, n_{n} \in S$, every vertex in $N\left[\left\{1, n_{n}\right\}\right]=\left\{1,2_{m}, 2_{k}, 2_{n},(n-1)_{n}, n_{n}, x\right\}$ is dominated by $S$. It is clear that $C(m, k, n)-N\left[\left\{1, n_{n}\right\}\right] \cong$ $P_{m-2} \cup P_{k-2} \cup P_{n-4}$ (see Figure 8).

In order to dominate the vertices in $C(m$, $k, n)-N\left[\left\{1, n_{n}\right\}\right]$, then $S-\left\{1, n_{n}\right\}$ must contain at least $\lceil(m-2) / 3\rceil+\lceil(k-2) / 3\rceil+\lceil(n-4) / 3\rceil$ vertices. We thus get $|S| \geq 2+\lceil(m-2) / 3\rceil+\lceil(k-2) / 3\rceil+$ $\lceil(n-4) / 3\rceil=2+\lceil(m-2) / 3\rceil+\lceil(k-2) / 3\rceil+\lceil(n-2) /$ 37. From Lemma 10 , we know that $C(m, k, n)$ contains a dominating set of order $1+\lceil(m-2) / 3\rceil+$ $\lceil(k-2) / 3\rceil+\lceil(n-2) / 3\rceil$. This contradicts the fact that $S$ is a $\gamma$ - set of $C(m, k, n)$.

(3) The proof is similar to that for 2 .

Lemma 12. Let $n, k, m \in \mathbb{N}-\{1,2\}$ and let $S$ be a $\gamma$ - set of $C(m, k, n)$ and $|S \cap\{1, x\}|=0$. Then, the following hold:

(1) If $m, k, n \equiv 1 \quad(\bmod 3)$, then either $2_{m}, m_{m} \in S$, $2_{k}, k_{k} \in S$, or $2_{n}, n_{n} \in S$.

(2) If $m \equiv 2 \quad(\bmod 3)$ and $k, n \equiv 1 \quad(\bmod 3)$, then $2_{m}, m_{m} \in S$.

(3) If $k \equiv 2(\bmod 3)$ and $m, n \equiv 1 \quad(\bmod 3)$, then $2_{k}, k_{k} \in S$

(4) If $n \equiv 2 \quad(\bmod 3)$ and $m, k \equiv 1 \quad(\bmod 3)$, then $2_{n}, n_{n} \in S$.

Proof. We give the proofs only for (1) and (2); the proofs of (3) and (4) are similar to that of (2).

(1) Let $m, k, n \equiv 1 \quad(\bmod 3)$. Suppose, by contrary, that neither $2_{m}, m_{m} \in S, 2_{k}, k_{k} \in S$ nor $2_{n}, n_{n} \in S$. So, in order to dominate 1 and $x$, we get $2_{q}, z_{z} \in S$ for some $q, z \in\{m, k, n\}$ such that $q \neq z$. There are nine cases for $q$ and $z$. We give the proof only for the case $q=m$ and $z=k$; the proofs of the other cases are similar. Since $2_{m}, k_{k} \in S$, every vertex in $N\left[\left\{2_{m}, k_{k}\right\}\right]=\left\{1,2_{m}, 3_{m},(k-1)_{k}, k_{k}, x\right\}$ is dominated by $S$. We check at once that $C(m, k, n)-N\left[\left\{2_{m}, k_{k}\right\}\right] \cong P_{m-3} \cup P_{k-3} \cup P_{n-1} \quad$ (see Figure 9).

In order to dominate the vertices in $C(m, k, n)-N\left[\left\{2_{m}, k_{k}\right\}\right]$, then $S-\left\{2_{m}, k_{k}\right\}$ must contain at least $\lceil(m-3) / 3\rceil+\lceil(k-3) / 3\rceil+\lceil(n-$ 1) $/ 3\rceil$ vertices. This gives $|S| \geq 2+\lceil(m-3) / 3\rceil+\lceil(k$ $-3) / 3\rceil+\lceil(n-1) / 3\rceil=2+\lceil(m-2) / 3\rceil+\lceil(k-2) / 3\rceil$ $+\lceil(n-2) / 3\rceil$. From Lemma 10, we know that $C(m, k, n)$ contains a dominating set of order $1+\lceil(m-2) / 3\rceil+\lceil(k-2) / 3\rceil+\lceil(n-2) / 3\rceil . \quad$ This contradicts the fact that $S$ is a $\gamma-$ set of $C(m, k, n)$.

(2) Let $m \equiv 2 \quad(\bmod 3)$ and $k, n \equiv 1 \quad(\bmod 3)$. On the contrary, suppose that $2_{m}, m_{m} \notin S$. In order to dominate 1 and $x$, then $2_{q}, z_{z} \in S$, for some $q, z \in\{m, k, n\}$ such that $q \neq z$ or $q=z \in\{k, n\}$. If $q \neq z$, then the proof is similar to that for 1 . Let $q=z \in\{k, n\}$. There are two cases for $q$ and $z$. We give the proof only for the case $q=z=k$; the proof of the case $q=z=n$ is similar. Since $2_{k}, k_{k} \in S$, every vertex in $N\left[\left\{2_{k}, k_{k}\right\}\right]=\left\{1,2_{k}, 3_{k},(k-1)_{k}, k_{k}, x\right\}$ is dominated by $S$. If $k \geq 5$, then $C(m, k, n)-N$ $\left[\left\{2_{k}, k_{k}\right\}\right] \cong P_{m-1} \cup P_{k-5} \cup P_{n-1}$ (see Figure 10).

In order to dominate the vertices in $C(m$, $k, n)-N\left[\left\{2_{k}, k_{k}\right\}\right]$, then $S-\left\{2_{k}, k_{k}\right\}$ must contain at least $\lceil(m-1) / 3\rceil+\lceil(k-5) / 3\rceil+\lceil(n-1) / 3\rceil$ vertices. We thus get $|S| \geq 2+\lceil(m-1) / 3\rceil+\lceil(k-5) / 3\rceil+$ $\lceil(n-1) / 3\rceil=3+\lceil(m-2) / 3\rceil+\lceil(k-2) / 3\rceil+\lceil(n-$ 2)/37. If $k<5$, then $k=4$. It is easily seen that $C(m, k, n)-N\left[\left\{2_{k}, k_{k}\right\}\right] \cong P_{m-1} \cup P_{n-1}$. In order to dominate the vertices in $C(m, k, n)-N\left[\left\{2_{k}, k_{k}\right\}\right]$, then $S-\left\{2_{k}, k_{k}\right\}$ must contain at least $\lceil(m-1) / 3\rceil+$ $\lceil(n-1) / 3\rceil$ vertices. This gives $|S| \geq 2+\lceil(m-1) / 3\rceil+$ $\lceil(n-1) / 3\rceil=2+\lceil(m-2) / 3\rceil+\lceil(k-2) / 3\rceil+\lceil(n-$ 


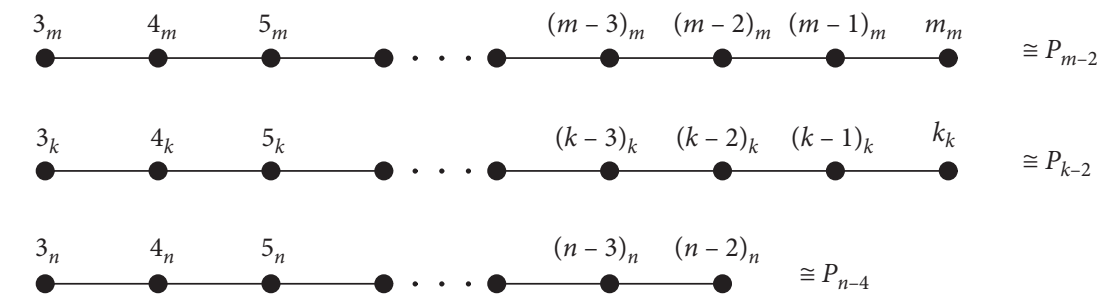

Figure 8: $C(m, k, n)-N\left[\left\{1, n_{n}\right\}\right]$.

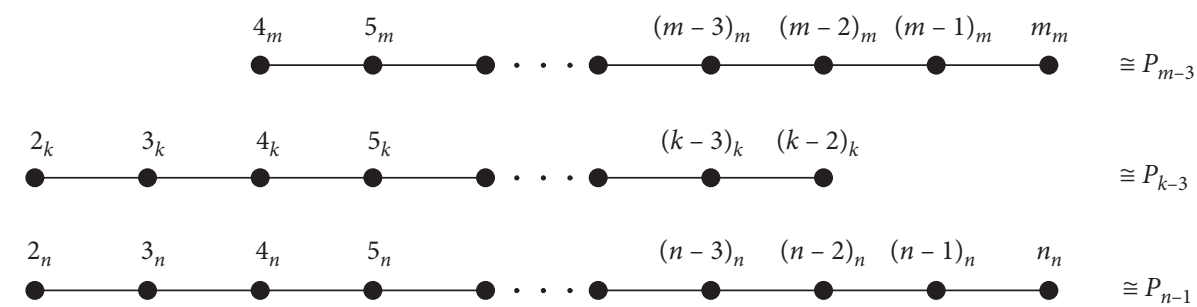

Figure 9: $C(m, k, n)-N\left[\left\{2_{m}, k_{k}\right\}\right]$.

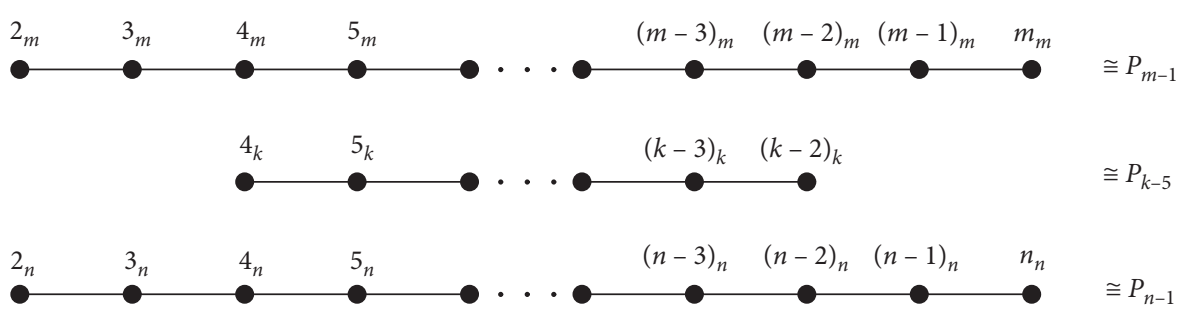

Figure 10: $C(m, k, n)-N\left[\left\{2_{k}, k_{k}\right\}\right]$.

2)/37. From Lemma 10 , we know that $C(m, k, n)$ contains a dominating set of order $1+\lceil(m-2) / 3\rceil$ $+\lceil(k-2) / 3\rceil+\lceil(n-2) / 3\rceil$. This contradicts the fact that $S$ is a $\gamma$ - set of $C(m, k, n)$.

Lemma 13. Let $n, k, m \in \mathbb{N}-\{1,2\}$ and let $S$ be a $\gamma$ - set of $C(m, k, n)$. Then, $|S \cap\{1, x\}|=1$ if one of the following holds:

(1) $m \equiv 1 \quad(\bmod 3)$ and $k, n \equiv 2 \quad(\bmod 3)$.

(2) $k \equiv 1 \quad(\bmod 3)$ and $m, n \equiv 2 \quad(\bmod 3)$.

(3) $n \equiv 1 \quad(\bmod 3)$ and $m, k \equiv 2 \quad(\bmod 3)$.
Proof. We give the proof only for the case $m \equiv 1 \quad(\bmod 3)$ and $k, n \equiv 2 \quad(\bmod 3)$; the proofs of the other cases are similar. Suppose, by contrary, that $|S \cap\{1, x\}| \neq 1$. By Lemma 11 (1), $|S \cap\{1, x\}|=0$. We thus get $1, x \notin S$. So, in order to dominate 1 and $x$, we get $2_{q}, z_{z} \in S$, for some $q, z \in\{m, k, n\}$. There are two cases: $q \neq z$ and $q=z$. Following in a same manner as the proof of Lemma 12 (2), we can obtain $|S| \geq 1+\lceil(m-2) / 3\rceil+\lceil(k-2) / 3\rceil+\lceil(n-2) / 3\rceil, \quad$ which contradicts the fact that $S$ is a $\gamma$-set of $C(m, k, n)$.

Theorem 1. Let $n, k, m \in \mathbb{N}-\{1,2\}, \quad D=\{m, k, n\}$, and $C=C(m, k, n)$. Then,

$$
\gamma(C)= \begin{cases}2+\left\lceil\frac{m-3}{3}\right\rceil+\left\lceil\frac{k-3}{3}\right\rceil+\left\lceil\frac{n-3}{3}\right\rceil & \text { if } y \equiv 0 \quad(\bmod 3) \text { for some } y \in D \text { or } m, k, n \equiv 2 \quad(\bmod 3), \\ 1+\left\lceil\frac{m-2}{3}\right\rceil+\left\lceil\frac{k-2}{3}\right\rceil+\left\lceil\frac{n-2}{3}\right\rceil & \text { if } m, k, n \equiv 0 \quad(\bmod 3) \text { and } y \equiv 1 \quad(\bmod 3) \text { for some } y \in D\end{cases}
$$

Case 1. $y \equiv 0 \quad(\bmod 3)$, for some $y \in D$ or $m, k, n \equiv 2 \quad(\bmod 3)$. By Lemma 9, $\gamma(C) \leq 2+\lceil(m-3) / 3\rceil+\lceil(k-3) / 3\rceil+\lceil(n-3) / 3\rceil$. We next prove that $\gamma(C) \geq 2+\lceil(m-3) / 3\rceil+\lceil(k-3) / 3\rceil$ $+\lceil(n-3) / 3\rceil$. Let $S$ be a $\gamma-$ set of $C$.
Case 1.1. $y \equiv 0 \quad(\bmod 3)$ for some $y \in D$. Without loss of generality, we can assume that $m \equiv 0 \quad(\bmod 3)$. In order to dominate 1 and $x$, there are sixteen cases, $u, v \in S$, for some $u \in\left\{1,2_{m}, 2_{k}, 2_{n}\right\}$ and $v \in\left\{x, m_{m}, k_{k}, n_{n}\right\}$. We give the proofs only for the 


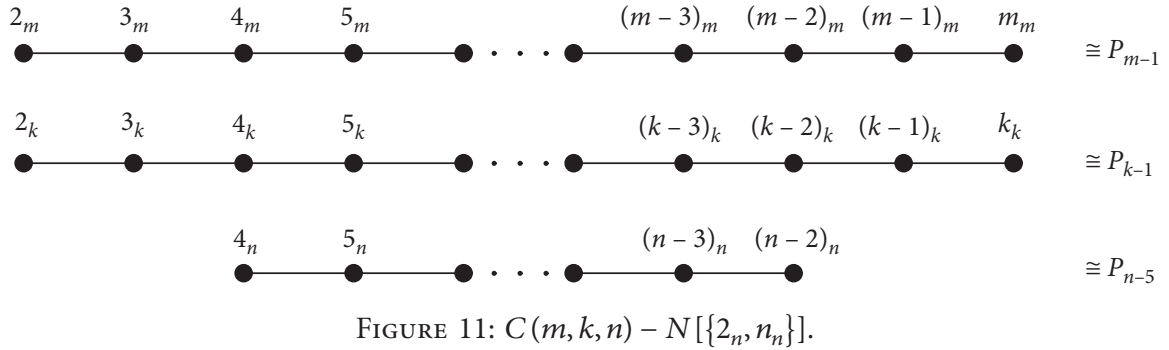

four cases $1, x \in S, \quad 1, m_{m} \in S, \quad 2_{m}, m_{m} \in S$, and $2_{m}, k_{k} \in S$; the proofs of the other cases are similar.

Case 1.1.1. 1, $x \in S$. Then, every vertex in $N[\{1, x\}]=$ $\left\{1,2_{m}, 2_{k}, 2_{n}, x, m_{m}, k_{k}, n_{n}\right\}$ is dominated by $S$. Thus, $C-N[\{1, x\}] \cong P_{m-3} \cup P_{k-3} \cup P_{k-3}$, so $\gamma(C)=|S| \geq 2$ $+\lceil(m-3) / 3\rceil+\lceil(k-3) / 3\rceil+\lceil(n-3) / 3\rceil$.

Case 1.1.2. $1, m_{m} \in S$. It follows that every vertex in $N\left[\left\{1, m_{m}\right\}\right]=\left\{1,2_{m}, 2_{k}, 2_{n},(m-1)_{m}, m_{m}, x\right\} \quad$ is dominated by $S$. If $m \geq 4$, then it is easy to check that $C-N\left[\left\{1, m_{m}\right\}\right] \cong P_{m-4} \cup P_{k-2} \cup P_{n-2}$. This gives $|S|$ $\geq 2+\lceil(m-4) / 3\rceil+\lceil(k-2) / 3\rceil+\lceil(n-2) / 3\rceil$. For $m$ $=3, C-N\left[\left\{1, m_{m}\right\}\right] \cong P_{k-2} \cup P_{n-2}$. It follows that $|S|$ $\geq 2+\lceil(k-2) / 3\rceil+\lceil(n-2) / 3\rceil$. Since $m \equiv 0 \quad(\bmod$ 3), $\gamma(C)=|S| \geq 2+\lceil(m-3) / 3\rceil+\lceil(k-3) / 3\rceil+\lceil(n$ $-3) / 3\rceil$.

Case 1.1.3. $2_{m}, m_{m} \in S$. Then, every vertex in $N\left[\left\{2_{m}\right.\right.$, $\left.\left.m_{m}\right\}\right]=\left\{1,2_{m}, 3_{m},(m-1)_{m}, m_{m}, x\right\}$ is dominated by $S$. We can see that $C-N\left[\left\{2_{m}\right.\right.$, $\left.\left.m_{m}\right\}\right] \cong P_{m-5} \cup P_{k-1} \cup P_{n-1}$ if $m \geq 5$, and $C-N$ $\left[\left\{2_{m}, m_{m}\right\}\right] \cong P_{k-1} \cup P_{n-1}$ otherwise. It follows that $|S| \geq 2+\lceil(m-5) / 3\rceil+\lceil(k-1) / 3\rceil+\lceil(n-1) / 3\rceil$ if $m \geq 5$, and $|S| \geq 2+\lceil(k-1) / 3\rceil+\lceil(n-1) / 3\rceil$ otherwise. Since $m \equiv 0 \quad(\bmod 3), \quad \gamma(C)=|S| \geq 2+$ $\lceil(m-3) / 3\rceil+\lceil(k-3) / 3\rceil+\lceil(n-3) / 3\rceil$.

Case 1.1.4. $2_{m}, k_{k} \in S$. We thus get every vertex in $N\left[\left\{2_{m}, k_{k}\right\}\right]=\left\{1,2_{m}, 3_{m},(k-1)_{k}, k_{k}, x\right\}$ is dominated by $S$. Since $C-N\left[\left\{2_{m}, k_{k}\right\}\right]$ $\cong P_{m-3} \cup P_{k-3} \cup P_{n-1}, \quad \gamma(C)=|S| \geq 2+\lceil(m-3) / 3\rceil+$ $\lceil(k-3) / 3\rceil+\lceil(n-3) / 3\rceil$.

Case $1.2 . m, k, n \equiv 2 \quad(\bmod 3)$. In order to dominate 1 and $x$, there are sixteen cases as above. In the same manner, we can prove that $\gamma(C)=2+$ $\lceil(m-3) / 3\rceil+\lceil(k-3) / 3\rceil+\lceil(n-3) / 3\rceil$.

Case $2 . m, k, n \equiv 0 \quad(\bmod 3)$ and $y \equiv 1 \quad(\bmod 3)$, for some $y \in D$. By Lemma $10, \gamma(C) \leq 1+\lceil(m-2) / 3\rceil+$ $\lceil(k-2) / 3\rceil+\lceil(n-2) / 3\rceil$. We next show that $\gamma(C) \geq 1+\lceil(m-2) / 3\rceil+\lceil(k-2) / 3\rceil+\lceil(n-2) / 3\rceil$.

Without loss of generality, we can assume that $m \equiv 1 \quad(\bmod 3)$. Let $S$ be a $\gamma-$ set of $C$. By Lemma 11
(1), $|S \cap\{1, x\}| \leq 1$. It follows that $|S \cap\{1, x\}|=1$ or $|S \cap\{1, x\}|=0$. Consider the following four cases.

Case 2.1. $k, n \equiv 1 \quad(\bmod 3)$. If $|S \cap\{1, x\}|=1$, then $1 \in S$ or $x \in S$. We give the proof only for the case $1 \in S$; the proof of the case $x \in S$ is similar. By Lemma 11 (2), $m_{m} \in S, k_{k} \in S$, or $n_{n} \in S$. Here, we will give the proof only for the case $m_{m} \in S$; the proofs of the other two cases are similar. Hence, $1, m_{m} \in S$. As in the proof of Case 1.1.2, $\gamma(C)=|S| \geq 2+\lceil(m-4) / 3\rceil+\lceil(k-2) / 3\rceil+$ $\lceil(n-2) / 3\rceil=1+\lceil(m-1) / 3\rceil+\lceil(k-2) / 3\rceil+\lceil(n-$ $2) / 3\rceil=1+\lceil(m-2) / 3\rceil+\lceil(k-2) / 3\rceil+\lceil(n-2) / 3\rceil$. If $|S \cap\{1, x\}|=0$, then $2_{m}, m_{m} \in S, 2_{k}, k_{k} \in S$, or $2_{n}, n_{n}$ $\in S$ by Lemma 12 (1). We give the proof only for the case $2_{m}, m_{m} \in S$; the proofs of the other two cases are similar. As in the proof of Case 1.1.3, we get that if $m \geq 5$, then $\quad \gamma(C)=|S| \geq 2+\lceil(m-5) / 3\rceil+\lceil(k-1) / 3\rceil+$ $\lceil(n-1) / 3\rceil=1+\lceil(m-2) / 3\rceil+\lceil(k-2) / 3\rceil+$ $\lceil(n-2) / 3\rceil$. If $m \leq 4$, then $m=4$. It follows that $\gamma(C)=$ $|S| \geq 2+\lceil(k-1) / 3\rceil+\lceil(n-1) / 3\rceil=1+\lceil(m-2) / 3\rceil+$ $\lceil(k-2) / 3\rceil+\lceil(n-2) / 3\rceil$.

Case 2.2. $k \equiv 1 \quad(\bmod 3)$ and $n \equiv 2 \quad(\bmod 3)$. If $|S \cap\{1, x\}|=1$, then $1 \in S$ or $x \in S$. We give the proof only for the case $1 \in S$; the proof of the case $x \in S$ is similar. By Lemma 11 (2), $m_{m} \in S$ or $k_{k} \in S$. Here, we will give the proof only for the case $m_{m} \in S$; the proof of the case $k_{k} \in S$ is similar. Hence, $1, m_{m} \in S$. As in the proof of Case 2.1, $\gamma(C)=|S| \geq 1+\lceil(m-2) / 3\rceil+$ $\lceil(k-2) / 3\rceil+\lceil(n-2) / 3\rceil$. If $|S \cap\{1, x\}|=0$, then $2_{n}, n_{n} \in S$ by Lemma 12 (4). Thus, every vertex in $N\left[\left\{2_{n}, n_{n}\right\}\right]=\left\{1,2_{n}, 3_{n},(n-1)_{n}, n_{n}, x\right\}$ is dominated by $S$. It is clear that $C-N\left[\left\{2_{n}, n_{n}\right\}\right]$ $\cong P_{m-1} \cup P_{k-1} \cup P_{n-5}$ (see Figure 11). In order to dominate the vertices in $C-N\left[\left\{2_{n}, n_{n}\right\}\right]$, then $S-\left\{2_{n}, n_{n}\right\}$ must contain at least $\lceil(m-1) / 3\rceil+\lceil(k-$ $1) / 3\rceil+\lceil(n-5) / 3\rceil$ vertices. This gives $|S| \geq 2+\lceil(m-$ $1) / 3\rceil+\lceil(k-1) / 3\rceil+\lceil(n-5) / 3\rceil=1+\lceil(m-2) / 3\rceil+$ $\lceil(k-2) / 3\rceil+\lceil(n-2) / 3\rceil$.

Case $2.3 . k \equiv 2 \quad(\bmod 3)$ and $n \equiv 1 \quad(\bmod 3)$. The proof is similar to that of Case 2.2. 
Case 2.4. $k, n \equiv 2 \quad(\bmod 3)$. By Lemma $13, \mid S \cap\{1$, $x\} \mid=1$. So, $1 \in S$ or $x \in S$. We give the proof only for the case $1 \in S$; the proof of the case $x \in S$ is similar. By Lemma $11(2), m_{m} \in S$. Following in a same manner as the proof of Case 2.1, we can obtain $\gamma(C)=|S|$ $\geq 1+\lceil(m-2) / 3\rceil+\lceil(k-2) / 3\rceil+\lceil(n-2) / 3\rceil$.

Summarizing, we get the domination number of amalgamations of cycles at connected subgraphs $C_{r} \triangleleft \triangleright C_{t}$.
From Lemmas 2-7 and Theorem 1, we then get the following theorem.

Theorem 2. Let $r, s, t \in \mathbb{N}, 3 \leq r \leq t, G=C_{r} \triangleleft \triangleright C_{t}$, and $D=$ $\mathrm{H}_{1} \cong_{f} \mathrm{H}_{2}$

$\{a, b, c\}$ where $a=r-s+1, b=s-1$, and $c=t-s+1$. Then

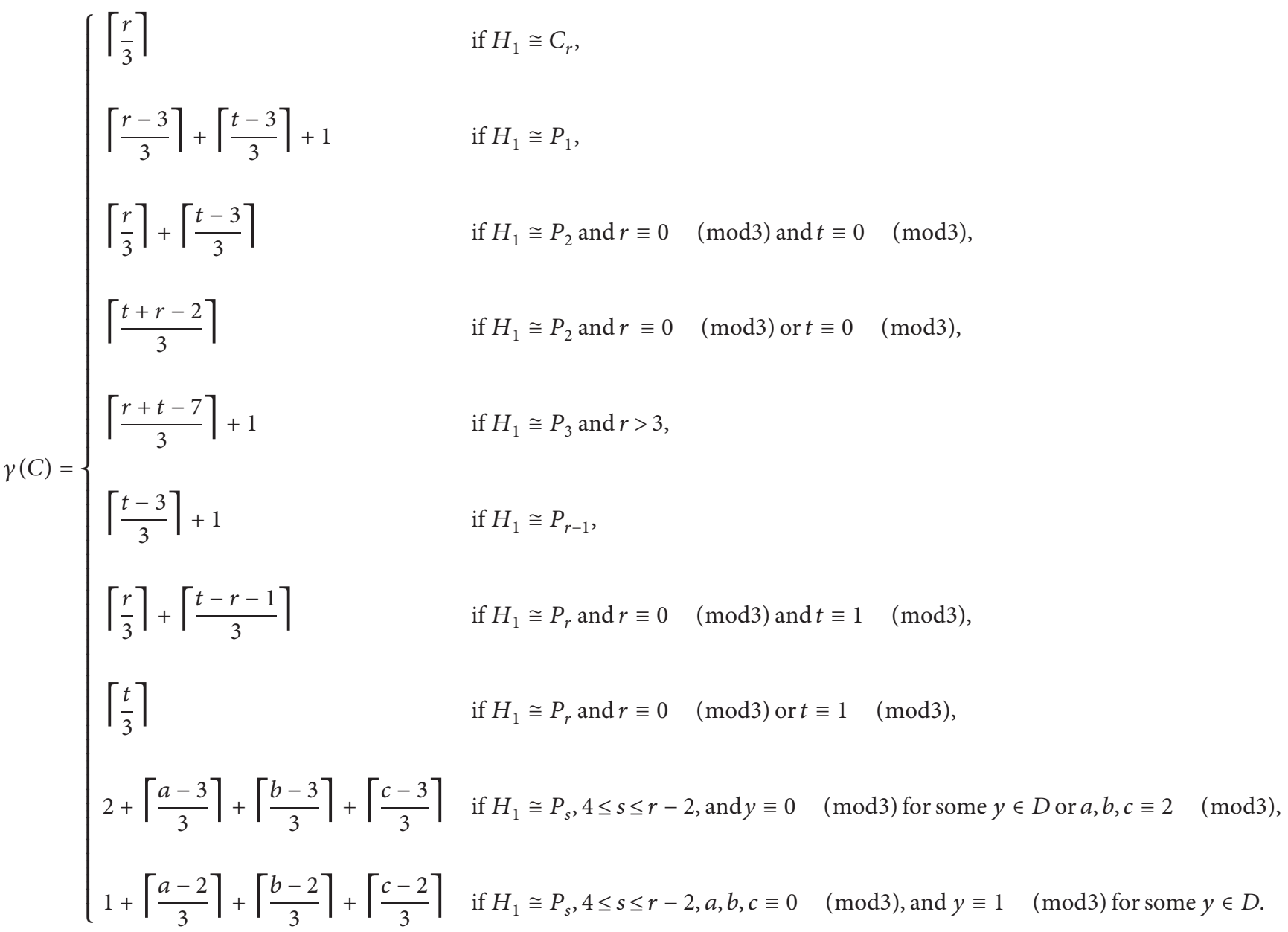

\section{Data Availability}

The data used to support the findings of this study are included within the article.

\section{Conflicts of Interest}

The authors declare that they have no conflicts of interest.

\section{Acknowledgments}

This research was supported by Chiang Mai University and Faculty of Science, Chiang Mai University, Thailand.

\section{References}

[1] C. Berge, Theory of Graphs and its Applications, Dunod, Paris, France, 1958.

[2] O. Ore, "Theory of graphs," American Mathematical Society, vol. 38, pp. 206-212, 1962.

[3] E. J. Cockayne and S. T. Hedetniemi, "Towards a theory of domination in graphs," Networks, vol. 7, no. 3, pp. 247-261, 1977.

[4] T. W. Haynes, S. T. Hedetniemi, and P. J. Slater, Fundamentals of Dominations in Graphs, Marcel Dekker, New York, NY, USA, 1998.

[5] S. Alikhani, Y. H. Peng, and K. A. M. Atan, "On the domination number of some graphs," International Mathematical Forum, vol. 3, pp. 1879-1884, 2008. 
[6] M. M. Bacolod and M. P. Baldado Jr., "Domination number of the acquaint vertex gluing of graphs," Applied Mathematical Sciences, vol. 8, pp. 8029-8036, 2014.

[7] T. T. Chelvam and G. Kalaimurugan, "Bounds for domination parameters in cayley graphs on dihedral group," Open Journal of Discrete Mathematics, vol. 2, pp. 5-10, 2012.

[8] R. Chérifi, S. Gravier, X. Lagraula, C. Payan, and I. Zigham, "Domination number of cross products of paths," Discrete Applied Mathematics, vol. 94, pp. 101-139, 1999.

[9] M. H. El-Zahar, S. M. Khamis, and K. M. Nazzal, "On the domination number of the cartesian product of the cycle of length n and any graph," Discrete Applied Mathematics, vol. 155 , no. 4, pp. 515-522, 2007.

[10] A. Frendrup, M. A. Henning, B. Randerath, and P. D. Vestergaard, "An upper bound on the domination number of a graph with minimum degree 2," Discrete Mathematics, vol. 309, pp. 639-646, 2009.

[11] S. Gravier and M. Mollard, "On domination numbers of cartesian product of paths," Discrete Applied Mathematics, vol. 80, pp. 247-250, 1997.

[12] S. Klaviar and N. Seifter, "Dominating Cartesian products of cycles," Discrete Applied Mathematics, vol. 59, pp. 129-136, 1995.

[13] A. V. Kostochka and C. Stocker, "A New Bound on the Domination Number of Connected Cubic Graph," Siberian Electronic Mathematical Reports, vol. 6, pp. 465-504, 2009.

[14] A. V. Kostochka and B. Y. Stodolsky, "An upper bound on the domination number of n-vertex connected cubic graphs," Discrete Mathematics, vol. 309, pp. 1142-1162, 2009.

[15] N. Murugesan and D. S. Nair, "The domination and independence of some cubic bipartite graphs," International Journal of Contemporary Mathematical Sciences, vol. 6, pp. 611-618, 2011.

[16] X. Zhang, J. Liu, and J. Meng, "Domination in lexicographic product graphs," Ars Combinatoria, vol. 101, pp. 251-256, 2011.

[17] C. Uiyyasathian, "Maximal-clique partitions," Ph. D thesis, University of Colorado at Denver, Denver, CO, USA, 2003.

[18] C. Promsakon and C. Uiyyasathian, "Chromatic numbers of glued graphs," Thai Journal of Mathematics, Special Issue (Annual Meeting in Mathematics), vol. 4, pp. 75-81, 2006.

[19] S. T. Hedetniemi and R. C. Laskar, Topics on Domination, Elsevier, Amsterdam, Netherlands, 1991. 